\title{
Fratura patológica associada a carcinoma pulmonar de células escamosas com metástases ósseas: um relato de caso
}

\author{
Pathological fracture associated with squamous cell lung cancer with bone metastasis: a \\ case report
}

\author{
Fractura patológica asociada a carcinoma de pulmón de celulas escamosas con \\ metastastos óseos: reporte de un caso
}

\begin{abstract}
Bruna Aparecida Carvalho Luiz ${ }^{1 *}$, Giliard Dário de Souza ${ }^{1}$, Anna Maria Pereira Meirelles Nicoliello", Gustavo Henrique Mendonça de Araújo ${ }^{1}$, Luiz Eduardo da Rocha Gonzaga ${ }^{1}$, Marcus Vinicius Monteiro Martins ${ }^{1}$, Iasmim Estela Costa ${ }^{1}$, Júlia Rezende Ribeiro ${ }^{1}$, Camila Souza de Oliveira Guimarães ${ }^{1}$.
\end{abstract}

\section{RESUMO}

Objetivo: Descrever um caso de fratura patológica de fêmur esquerdo em paciente com câncer de pulmão de células escamosas metastático. Detalhamentos de Caso: ALC, 72 anos, sexo masculino, ex-tabagista, compareceu à Unidade Básica de Saúde com queixa de dor em perna esquerda. Sem queixas respiratórias, o paciente apresentava lesão suspeita em parede torácica que foi submetida ao exame complementar, além de radiografia do membro inferior esquerdo para avaliação. Com resultado de exames de imagem, o paciente foi encaminhado à unidade de referência oncológica e foi realizado biópsia da lesão torácica. $A$ análise histopatológica evidenciou carcinoma pulmonar de células não pequenas metastático, com expressão do marcador P40, indicativo de carcinoma de células escamosas. Apresentava na cintilografia múltiplas metástases, evoluindo com fratura patológica em fêmur esquerdo enquanto aguardava início do tratamento oncológico. Considerações finais: Apesar dos avanços terapêuticos, a mortalidade por esse subtipo de câncer ainda é significativa e correlacionada principalmente ao estadiamento do câncer ao diagnóstico. Assim, a identificação precoce e a prevenção primária no combate sistemático ao tabagismo são as principais ferramentas para reduzir a incidência e, consequentemente, a morbimortalidade associada à doença.

Palavras-chave: Carcinoma de células escamosas, Carcinoma escamoso, Cuidado paliativo, Oncologia.

\begin{abstract}
Objective: To describe a case report of a pathological fracture of the left femur in a patient with metastatic squamous cell lung cancer. Case Details: ALC, 72 years old, male, ex-smoker, attended the Basic Health Unit complaining of pain in his left leg. Without respiratory complaints, the patient had a suspicious lesion in the chest wall that underwent a complementary examination, in addition to radiography of the left lower limb for evaluation. As a result of imaging exams, the patient was referred to the oncology referral unit and a biopsy was performed in the chest wall lesion. Histopathological analysis showed metastatic non-small cell lung carcinoma, with expression of the P40 marker, indicative of squamous cell carcinoma. The scintigraphy showed multiple metastases, evolving with a pathological fracture in the left femur while waiting for cancer treatment to begin. Final considerations: Despite therapeutic advances, mortality from this cancer subtype is still significant, and is mainlyrelated with cancer stage at diagnosis. Thus, early identification and primary prevention in the systematic fight against smoking are the main tools to reduce the incidence and, consequently, the morbidity and mortality associated with the disease.
\end{abstract}

Key words: Squamous cell carcinoma, Squamous carcinoma, Palliative care, Oncology.

1Universidade Federal de Lavras (UFLA). Lavras - MG. *E-mail: brunamedufla@gmail.com 


\section{RESUMEN}

Objetivo: Describir el caso clínico de una fractura patológica de fémur izquierdo en un paciente con cáncer de pulmón de células escamosas metastásico. Detalle del caso: ALC, 72 años, varon, exfumador, acudió a la Unidad Básica de Salud por dolor en pierna izquierda. Sin quejas respiratorias, el paciente tenía una lesión sospechosa en la pared torácica, a la que se le realizó un examen complementario, además de una radiografía de miembro inferior izquierdo para su evaluación. Como resultado de los exámenes de imagen, se derivó al paciente a la unidad de derivación de oncología y se le realizó una biopsia en la pared torácica. El análisis histopatológico mostró carcinoma de pulmón de células no pequeñas metastásico, con expresión del marcador P40, indicativo de carcinoma de células escamosas. La gammagrafía mostró múltiples metástasis, evolucionando con una fractura patológica en el fémur izquierdo a la espera del inicio del tratamiento oncológico. Consideraciones finales: A pesar de los avances terapéuticos, la mortalidad por este subtipo de cáncer sigue siendo significativa y se correlaciona principalmente con la estadificación del cáncer en lo momento del diagnóstico. Así, La identificación precoz y la prevención primaria en la lucha sistemática contra el tabaquismo son las principales herramientas para reducir la incidencia y, en consecuencia, la morbilidad y mortalidad asociadas a la enfermedad.

Palabras clave: Carcinoma de células escamosas, Carcinoma escamoso, Cuidados paliativos, Oncología.

\section{INTRODUÇÃO}

O câncer é um dos principais problemas de saúde pública do mundo, e tem se tornado cada vez mais prevalente na população. O envelhecimento populacional, o crescimento demográfico e as exposições aos fatores de risco são fatores contribuintes para esse aumento de incidência e mortalidade por câncer. Para 2020, o Instituto Nacional do Câncer José Alencar Gomes da Silva (INCA) estimou a ocorrência de 625 mil novos casos de câncer no Brasil (MINISTÉRIO DA SAÚDE, 2019).

O câncer de pulmão é um dos tipos com maior incidência e mortalidade no Brasil. As neoplasias primárias de pulmão corresponderam a aproximadamente 28 mil novos casos em 2016, sendo a sobrevida em 5 anos desses pacientes em torno de $18 \%$ (ALLEMANI C, et al., 2015).

O fator de risco mais associado ao desenvolvimento de câncer de pulmão é a exposição prolongada ao tabaco, seja ativa ou passivamente, e está presente em até $85 \%$ dos acometidos. Outros fatores associados ao desenvolvimento da doença são relacionados a exposição ocupacional principalmente a asbesto, sílica e urânio; outros determinantes são fatores genéticos e a poluição do ar (MINISTÉRIO DA SAÚDE, 2019; ALLEMANI C, et al., 2015; THUN MJ, et al., 2008).

Um dos maiores desafios no enfretamento à doença, além dos obstáculos encontrados na prevenção primária com a educação em saúde, está na dificuldade de estabelecer o diagnóstico precoce. Muitas vezes oligossintomático, a identificação desse tipo de câncer pode ser tardia, sendo esse um dos principais fatores determinantes para o prognóstico não favorável (ALLEMANI C, et al., 2015). Assim, a maioria dos casos são diagnosticados tardiamente, com o paciente já apresentando doença avançada (THUN MJ, et al., 2008).

O câncer pulmonar de células não pequenas (CPCNP) corresponde ao subtipo com diagnóstico mais tardio e com menor taxa de sobrevida (ARAUJO LH, et al., 2018). Pode-se dizer que o acesso a exames de imagem complementares(como a tomografia computadorizada)ainda é difícil em parte do país, sendo esse acesso ainda mais complicado quando tratamos de procedimentos mais invasivos (como a broncoscopia), fato que pode contribuir para o atraso no diagnóstico (ARAUJO LH, et al., 2018).

A depender do estadiamento, a conduta terapêutica pode ser dividida em curativa ou paliativa, sendo que, infelizmente, apenas uma pequena taxa dos indivíduos é submetida a tratamento curativo, devido ao diagnóstico em fase avançada (ARAUJO LH, et al., 2018).

Diante dos altos números de diagnósticos tardios, muito se tem discutido sobre a implantação de programas de rastreamento mais efetivos e de educação em saúde, que sejam capazes de conscientizar a 
população quanto aos fatores de risco, aos possíveis sinais e sintomas e à importância de assistência médica efetiva, atualizada e constante (ARAUJO LH, et al., 2018).

Um protocolo efetivo para rastreamento de câncer de pulmão teria o potencial de detectar estágio iniciais da doença, com o objetivo de tratamento precocemente, redução da mortalidade, aumento da sobrevida e melhora da qualidade de vida. Os métodos diagnósticos tem sido avaliados e em uma revisão sistemática, foi demonstrado que o rastreamento com radiografia de tórax não apresentou nenhum efeito sobre a mortalidade associada ao câncer de pulmão, impossibilitando sua elegibilidade (TANG X, et al., 2019; USMAN A, et al., 2016).

Por sua vez, o rastreamento com tomografia computadorizada de tórax foi capaz de reduzir a mortalidade. Todavia, esse método elevou os índices de falsos positivos e excesso de diagnósticos, com pacientes sendo submetidos desnecessariamente a intervenções invasivas e com complicações decorrentes dessa, inclusive morte (TANG X, et al., 2019; USMAN A, et al., 2016).

Destacam-se os seguintes desfechos: infecção, sangramento, hemotórax, pneumotórax, colapso pulmonar, dor, arritmias cardíacas, complicações tromboembólicas e necessidade de hospitalização (USMAN A, et al., 2016). Dessa forma, o rastreamento submeteria pessoas saudáveis a riscos que não compensariam os benefícios alcançados para os pacientes com a doença.

Logo, diante de uma doença de grande importância na saúde pública, com elevada morbimortalidade, e fortemente associada a um fator de risco que pode ser combatido com medidas de saúde pública, torna-se importante a discussão sobre medidas que contribuam para a prevenção e para o diagnóstico precoce do câncer de pulmão.

O presente relato tem por objetivo descrever o caso de um paciente diagnosticado com câncer de pulmão metastático que evoluiu com fratura patológica de fêmur, correlacionando os dados do caso com a literatura.

\section{DETALHAMENTO DO CASO}

ALC, 72 anos, sexo masculino, compareceu à Unidade Básica de Saúde queixando dor em membro inferior esquerdo, com dificuldade para deambulação. Referiu já ter buscado atendimento em Unidade de Pronto Atendimento, onde fora prescrito anti-inflamatórios e analgésicos, mas sem melhora da dor.

Relatou ser ex-tabagista, tendo cessado o hábito há mais de 20 anos. Negou outras comorbidades. Ao exame físico apresentava lesão nodular, endurecida e fixada a planos profundos em região esterno-clavicular (Figura 1) e dor à mobilização do membro inferior esquerdo. Foi solicitada radiografia de quadril (Figura 2), exames laboratoriais de rotina e ressonância magnética do tórax para avaliação (Figura 3 e 4).

Diante dos achados, por suspeita de câncer em estágio avançado, o paciente foi encaminhado para unidade de referência em oncologia, onde foi realizada biópsia da lesão esterno-clavicular. $O$ exame anatomopatológico teve como conclusão neoplasia maligna indiferenciada infiltrando tecido fibromuscular, sendo solicitada complementação com estudo imunohistoquímico.

A imunohistoquímica evidenciou carcinoma de células não pequenas metastático, com expressão do marcador P40, indicativo de carcinoma de células escamosas. Paciente apresentou piora da dor em fêmur esquerdo, sendo necessária a utilização de metadona 10mg BID.

No entanto, mesmo com o uso da medicação, continuou apresentando quadro álgico e mobilidade reduzida do membro, ficando mais restrito ao leito. Pela cintilografia óssea, foram evidenciados focos metastáticos em região esternal, coluna cervical anterior, coluna torácica média e fêmur esquerdo.

Enquanto aguardava o início da quimioterapia/radioterapia em serviço de referência, o paciente apresentou fratura patológica do fêmur esquerdo (Figura 5), sendo encaminhado para correção cirúrgica em centro de referência. 
Figura 1 - Lesão nodular em região esterno-clavicular.

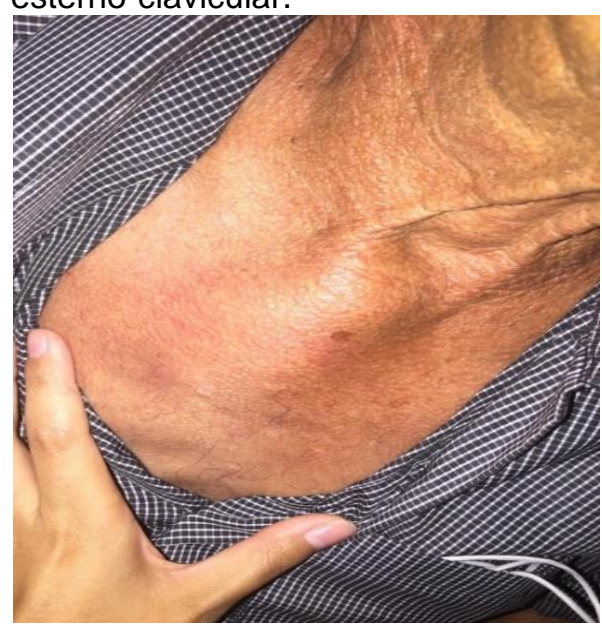

Fonte: Luiz BAC, et al., 2020.

Figura 2 - Lesão radioluscente de aspecto inespecífico em fêmur esquerdo.

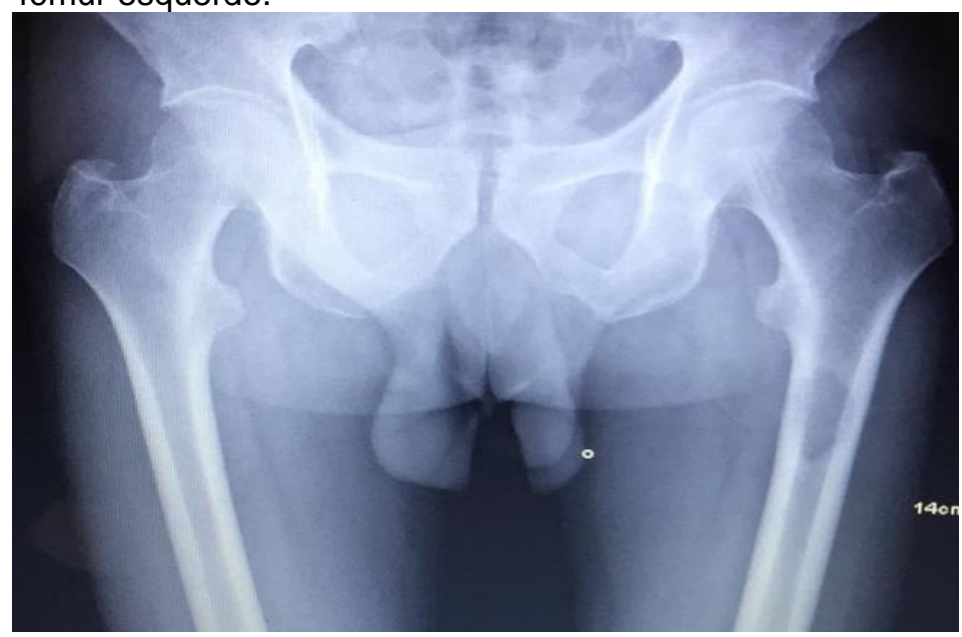

Fonte: Luiz BAC, et al., 2020.

Figura 3 - Ressonância Magnética de Tórax evidenciando lesão de aspecto neoplásico acometendo o manúbrio esternal.

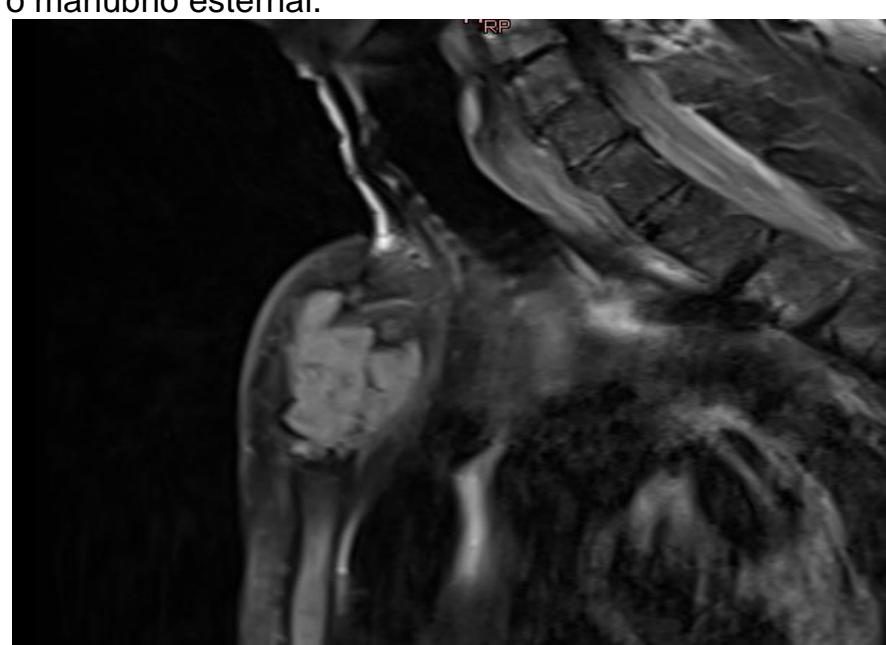

Fonte: Luiz BAC, et al., 2020. 
Figura 4 - Nódulo sólido de margens irregulares localizado no segmento anterior do lobo superior do pulmão direito.

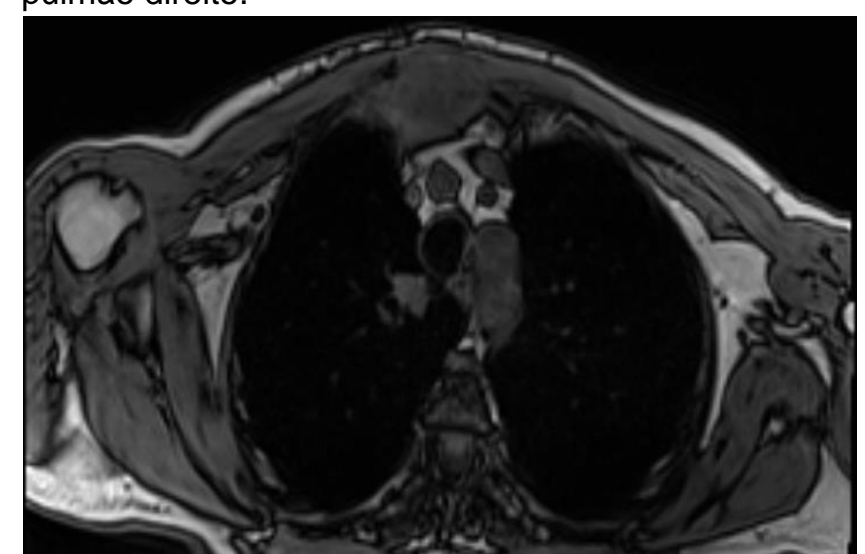

Fonte: Luiz BAC, et al., 2020.

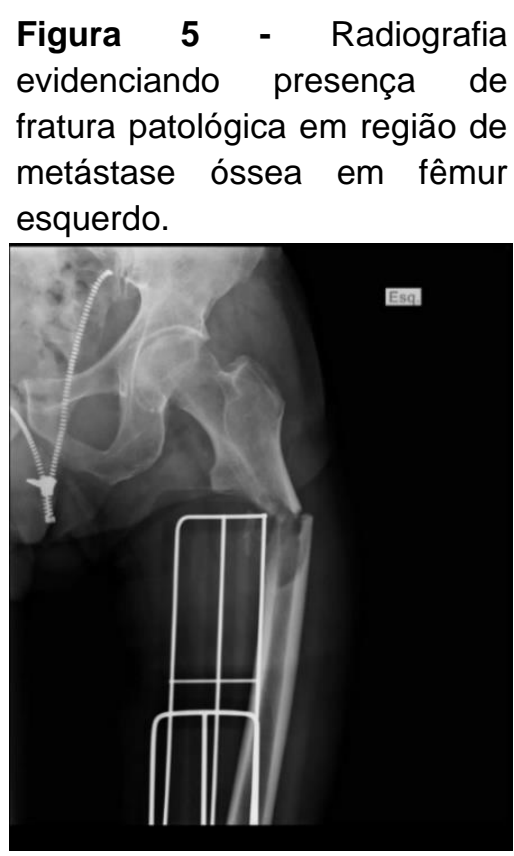

Fonte: Luiz BAC, et al., 2020.

\section{DISCUSSÃO}

O tabagismo é um importante fator de risco relacionado ao câncer de pulmão e ainda mais relevante do que a quantidade de cigarros fumados, é a duração do hábito (THUN MJ, et al., 2008.) Por ser um fator de risco modificável, a implementação de políticas de saúde pública com ações de educação em saúde que visem à redução do tabagismo, é de extrema importância.

No Brasil, algumas medidas já foram implementadas e trouxeram resultados benéficos: estudos conduzidos no país indicam que tanto a prevalência do tabagismo como as mortes com ele relacionadas diminuíram aproximadamente 50\% (MENDONÇA GAS, 2012). Dentre as estratégias das políticas brasileiras antitabaco estão ações de educação, com alertas em meios de comunicação e rótulos de embalagens de cigarros; leis de proibição do tabagismo em ambientes públicos e impostos mais altos sobre os produtos do tabaco (ARAUJO LH, et al., 2018).

Considerando a letalidade desse tipo de câncer e a sua incidência, muito tem-se discutido sobre programas de rastreamento para tal neoplasia. Alguns protocolos são divergentes em termos de conduta, mas a grande 
maioria concorda que, com os dados disponíveis atualmente, ainda não há indicação de incorporar programas de rastreamento na saúde pública. A radiografia de tórax, apesar de acessível, não gerou impacto na mortalidade (USMAN A, et al., 2016). Já o rastreamento por tomografia helicoidal reduz a mortalidade por câncer pulmonar (THE NACIONAL LUNG SCREENING TRIAL RESEARCH, 2020).

Apesar desse desfecho positivo, a tomografia foi associada a aumento no número de sobre diagnósticos (com aumento de falsos-positivos e diagnóstico de cânceres que não teriam se tornado sintomáticos) e o risco de outros cânceres induzidos por radiação, que por se tratar de uma manifestação a longo prazo não pôde ser avaliada nos estudos, sendo necessário novos estudos para avaliação (MINISTÉRIO DA SAÚDE, 2010; DUBEY AK, 2016; AZAR FE, et al., 2017; THE NACIONAL LUNG SCREENING TRIAL RESEARCH, 2020).

Além disso, para implementação desse programa de rastreamento na saúde pública também seria necessário avaliar criteriosamente o custo-benefício, já que a triagem não inclui apenas gastos com o exame, mas também com o tratamento e com o acompanhamento dos pacientes, sendo importante que, ao se decidir pela implementação do rastreio, os critérios de elegibilidade, a frequência da triagem e a interpretação dos resultados sejam bem estabelecidos previamente (THE NACIONAL LUNG SCREENING TRIAL RESEARCH, 2020).

Dados de estudos de acompanhamento a longo prazo dos pacientes submetidos a rastreio tomográfico poderão fornecer evidências mais conclusivas sobre a eficácia e aplicabilidade do teste, com delineamento de protocolo padronizado que melhore a precisão diagnóstica e reduza os potenciais danos associados (USMAN A, et al., 2016).

Em relação às manifestações clínicas, o espectro de sinais e sintomas é muito amplo. Os pacientes podem se apresentar em consulta desde assintomáticos a um quadro rico e típico. Em geral, o sintoma mais comum e que ocorre de forma mais precoce é a tosse (cerca de 75\%) (HIRANO H, et al., 2015). Entretanto, é o sintoma mais inespecífico, visto que os pacientes normalmente já são pneumopatas e fumantes, o que gera fator de confusão no diagnóstico e muitas vezes posterga a investigação em relação ao tumor.(HIRANO H, et al., 2015; GRECO MT, et al., 2014).

Segundo o Colégio Brasileiro de Cirurgiões, as neoplasias pulmonares podem ainda ser reconhecidas pelas metástases como manifestações iniciais da doença, fato considerado, felizmente, raro (GRECO MT, et al., 2014). Entretanto, essa não foi a casuística encontrada no paciente do caso. As metástases ósseas ocorrem entre $10 \%$ a $35 \%$ dos casos, sendo que, nesses,são características a dor localizada e a limitação de movimentos da região acometida (GRECO MT, et al., 2014). Os locais mais comuns de acometimento são arcos costais, corpos vertebrais e ossos longos proximais.

O paciente ALC não tinha nenhuma queixa respiratória, mas apresentava uma lesão suspeita em parede torácica, em região esternal, que suscitou a investigação. Devido à alta agressividade do tumor, a análise histopatológica foi inconclusiva, sendo necessária a realização da imunohistoquímica para elucidação diagnóstica e definição do caso.

No caso relatado, chama a atenção o fato de o paciente apresentar fratura patológica, quadro reservado a apenas 1 a 2\% dos casos de câncer pulmonar (OLIVEIRA MBR, et al., 2018). A correção cirúrgica pode ser realizada em casos de fratura já estabelecida, ou mesmo em casos de iminência de fratura patológica. As indicações de abordagem cirúrgica são controversas e geralmente são estabelecidas pelo serviço de oncologia. No entanto, as indicações mais aceitas consideram o estado geral de saúde do paciente, o tempo de sobrevida, a carga suportada pelo osso afetado, o estoque ósseo remanescente e o prognóstico do paciente quanto à mobilidade.

No caso das fraturas patológicas iminentes, um escore de Mirels acima de 8 pontos pode ser associado ao julgamento clínico para suportar a indicação da abordagem cirúrgica (MEOHAS W, et al., 2005). Outras metástases descritas para o câncer de pulmão são a cerebral e a hepática, que muitas vezes são descobertas apenas quando sintomáticas (AZAR FE, et al., 2017; OLIVEIRA MBR, et al., 2018). 
Esses dados reforçam a importância do diagnóstico precoce, a fim de evitar complicações e reduzir a morbimortalidade da doença. Entretanto, sabe-se da dificuldade de se realizar o rastreamento com os meios atualmente disponíveis, tendo em vista que isso implicaria na exposição de pessoas sem a doença aos riscos da submissão a exames complementares de alta complexidade.

Dessa forma, ressalta-se a importância da prevenção primária como ferramenta essencial na redução da incidência dos cânceres de pulmão, com enfoque nas estratégias de promoção à saúde e abandono do tabagismo. O impacto das políticas públicas de controle do tabagismo na redução da incidência e da mortalidade por câncer de pulmão é significativo, sobretudo nos países desenvolvidos, mas também já foi demonstrado no Brasil (MENDONÇA GAS, 2012).

A partir da década de 1980, as políticas públicas de redução do tabagismo no Brasil têm obtido bons resultados. A comparação dos resultados da Pesquisa Nacional de Saúde e Nutrição, realizada em 1989, com os dados obtidos em 2008 pela Pesquisa Especial sobre Tabagismo, evidencia que durante esse período houve uma queda significativa na prevalência de tabagismo, tanto em homens quanto em mulheres (MENDONÇA GAS, 2012).

No entanto, é importante atentar-se para a diferença significativa entre os dados de prevalência de tabagismo de acordo com a escolaridade e com o nível socioeconômico. Dessa diferença, surge a percepção de que as populações menos esclarecidas e de baixa renda necessitam de medidas especiais e de estratégias que ampliem o impacto dessas políticas que vêm sendo desenvolvidas (MENDONÇA GAS, 2012).

É importante salientar que o tabagismo também é fator de risco importante para outras doenças, como doença pulmonar obstrutiva crônica, além de outros tipos de câncer, como de laringe, de esôfago, de boca e de faringe (GUERRA MR, et al., 2005; MENDONÇA GAS, 2012). Dessa forma, ressalta-se ainda mais a importância da intensificação das ações de prevenção e redução do tabagismo no Sistema Único de Saúde, tendo a Atenção Primária em Saúde como principal aliada, com o objetivo de assegurar e aprimorar os avanços obtidos pelas políticas implementadas.

Quanto ao diagnóstico precoce, é possível que uma seleção criteriosa de pacientes que se beneficiariam da investigação com exames complementares amenize os riscos envolvidos. No entanto, essa possibilidade carece de estudos que avaliem sua eficácia e efetividade, observando o impacto disso na morbimortalidade pela doença.

Por se tratar de uma temática de grande importância no cenário de saúde pública e, ainda, apresentar um fator de risco passível de intervenção através de Políticas de Saúde, torna-se extremamente necessário o debate sobre câncer pulmonar. O diagnóstico ainda se apresenta como o maior desafio no enfrentamento dessa patologia, já que grande parte dos pacientes ainda são diagnosticados tardiamente. Corroborando ainda mais para a dificuldade diagnóstica, programas de rastreamento atuais não conseguiram demonstrar benefícios que superem os riscos, os quais são significativos quando pessoas saudáveis são submetidas ao rastreio.

Por fim, vale apontar que, apesar do avanço terapêutico das neoplasias, a mortalidade por esse subtipo de câncer ainda é significativa, o que demonstra a importância da prevenção primária no combate sistemático ao tabagismo e coloca essa prerrogativa como uma importante ação de saúde pública para reduzir a incidência e, consequentemente, a mortalidade por essa patologia.

\section{REFERÊNCIAS}

1. ALLEMANI C, et al. Global surveillance of cancer survival 1995-2009: analysis of individual data for $25: 676: 887$ patients from 279 population-based registries in 67 countries (concord-2). The Lancet, 2015; 385(9972): 977-1010.

2. ARAUJO LH, et al. Câncer de Pulmão no Brasil. Jornal Brasileiro de Pneumologia, 2018; 44(1): 55-64.

3. AZAR FE, et al. Cost-effectiveness of lung cancer screening and treatment methods: a systematic review of systematic reviews. Bmc Health Services Research, 2017; 17(1): 1-9.

4. BRASIL Ministério da Saúde. Caderno de Atenção Primária: rastreamento. 29. ed. Brasília: MS, 2010; 95 p. 
5. BRASIL Ministério da Saúde. Estimativa 2020: Incidência de Câncer no Brasil. Instituto Nacional de Câncer José Alencar Gomes da Silva, 2019; 120p.

6. DUBEY AK, et al. Epidemiology of lung cancer and approaches for its prediction: a systematic review and analysis. Chinese Journal Of Cancer, 2016; 35(1): 1-12.

7. GRECO MT, et al. Quality of Cancer Pain Management: an update of a systematic review of undertreatment of patients with cancer. Journal Of Clinical Oncology, 2014; 32(36): 4149-4154.

8. GUERRA MR, et al. Risco de câncer no Brasil: tendências e estudos epidemiológicos mais recentes. Revista Brasileira de Cancerologia, 2005; 51(3): 227-234.

9. HIRANO H, et al. Survivin expression in lung cancer: association with smoking, histological types and pathological stages. Oncology Letters, 2015; 10(3): 1456-1462.

10. MENDONÇA GAS. Câncer de pulmão e as tendências atuais do tabagismo no Brasil.Cad. Saúde Pública, 2012; 28(9): 1620-1621.

11. MEOHAS W, et al. Metástase óssea: revisão da literatura. Revista Brasileira de Cancerologia, 2005; 51(1): 43-47.

12. OLIVEIRA MBR, et al. Pathological fractures due to bone metastases from lung cancer: risk factors and survival. Acta ortopedica brasileira, 2018; 26(6): 388-393.

13. TANG X, et al. Low-dose CT screening can reduce cancer mortality: A meta-analysis. Rev. Assoc. Med. Bras, 2019; 65(12): 1508-1514.

14. THE NATIONAL LUNG SCREENING TRIAL RESEARCH TEAM. Reduced lung-cancer mortality with low-dose computed tomographic screening. New England Journal of Medicine, 2011; 365(5): 395-409.

15. THUN MJ, et al. Lung cancer occurrence in never-smokers: an analysis of 13 cohorts and 22 cancer registry studies. PLoS Med, 2008;5(9):9.

16. USMAN A, MILLER J, PEIRSON L et al. Screening for lung cancer: a systematic review and meta-analysis. Preventive medicine, 2016; 89: 301-31. 